\title{
Revista Colombiana de

\section{La paradoja de la obesidad y su relación con la aptitud cardiorrespiratoria en pacientes con insuficiencia cardiaca}

\author{
Lisbeth Triviño ${ }^{a}$, Juan C. Ávila ${ }^{\mathrm{b}}$ y Robinson Ramírez-Vélez ${ }^{\mathrm{c}, *}$ \\ a Programa de Especialización en Rehabilitación Cardiopulmonar, Fundación Universitaria del Área Andina, Pereira, Colombia \\ b Programa de Fisioterapia, Fundación Universitaria María Cano Extensión Cali, Santiago de Cali, Colombia \\ c Grupo Ejercicio Físico y Deporte, Universidad Manuela Beltrán, Facultad de Salud, Bogotá, Colombia
}

Recibido el 10 de marzo de 2014; aceptado el 4 de mayo de 2015

Disponible en Internet el 8 de julio de 2015

\author{
PALABRAS CLAVE \\ Insuficiencia \\ cardiaca; \\ Obesidad; \\ Ejercicio
}

\begin{abstract}
Resumen
Antecedentes: A pesar de que el índice de masa corporal se asocia con mejores resultados en la insuficiencia cardiaca establecida, el impacto de la aptitud cardiorrespiratoria sobre la paradoja de la obesidad es menos claro.

Objetivo: Evaluar la relación entre el estado nutricional por el índice de masa corporal y los niveles de aptitud cardiorrespiratoria en pacientes con insuficiencia cardiaca.

Materiales y métodos: Se realizó un estudio transversal y descriptivo en 40 sujetos con insuficiencia cardiaca (edad 66,8 $\pm 11,4$ años; índice de masa corporal 27,4 $\pm 4,8 \mathrm{~kg} \cdot \mathrm{m}^{-1}$; fracción de eyección del ventrículo izquierdo $40,5 \pm 8,3 \%$ ). Se evaluó el índice de masa corporal como indicador de obesidad y el riesgo se clasificó según valores de referencia internacionales. La aptitud cardiorrespiratoria medida con el test de caminata de seis minutos y los valores del consumo de oxigeno por VO2max, la distancia y el numero de pasos alcanzados se agruparon y compararon por los valores de referencia del indice de masa corporal.

Resultados: En los sujetos con índice de masa corporal $\geq 25 \mathrm{~kg} \cdot \mathrm{m}^{-1}$, se observaron correlaciones negativas en la distancia alcanzada en el test de caminata de $6 \mathrm{~min}(r h o=-0,50)$, el número de pasos de dicho test $(r h o=-0,45)$, el $\mathrm{VO}_{2 \operatorname{máx}}(r h o=-0,49)$ y la fracción de eyección del ventrículo izquierdo ( $r h o=-0,32)$.

Conclusiones: Este estudio sugiere que los valores mayores de índice de masa corporal «paradoja de la obesidad», no se relacionan con un mejor nivel cardiorrespiratorio por $\mathrm{VO}_{2 \text { máx }}$. (c) 2014 Sociedad Colombiana de Cardiología y Cirugía Cardiovascular. Publicado por Elsevier España, S.L.U. Este es un artículo Open Access bajo la licencia CC BY-NC-ND (http://creativecommons.org/licenses/by-nc-nd/4.0/).
\end{abstract}

\footnotetext{
* Autor para correspondencia.

Correo electrónico: robin640@hotmail.com (R. Ramírez-Vélez).
} 


\section{KEYWORDS}

Heart failure;

Obesity;

Exercise

\section{The paradox of obesity and its relationship to cardiorespiratory fitness in patients with heart failure}

\begin{abstract}
Background: Although body mass index (BMI) is associated with improved outcomes in established heart failure (HF), the impact of cardiorespiratory fitness on the obesity paradox is less clear.

Objective: The aim of this study was to evaluate the relationship between body mass index and cardiorespiratory fitness in terms of $\mathrm{VO}_{2}$ max in patients with $\mathrm{HF}$.

Material and methods: A cross-sectional descriptive study was conducted on 40 patients with HF (age $66.8 \pm 11.4$ years, BMI $27.4 \pm 4.8 \mathrm{~kg} \cdot \mathrm{m}^{-1}$, LVEF $40.5 \pm 8.3 \%$ ). BMI was measured as an indicator of obesity and the risk was classified according to international benchmarks. Cardiorespiratory fitness, measured using the 6-min walk test (6'WT), oxygen consumption by $\mathrm{VO}(2) \mathrm{max}$, the distance and the number of steps, were grouped and compared with the body mass index reference values.

Results: In subjects with $\mathrm{BMI} \geq 25 \mathrm{~kg} \cdot \mathrm{m}^{-1}$, negative correlations were observed in the distance by 6'WT $(r h o=-0.50)$, number of steps $(r h o=-0.45)$, VO $(2) \max (r h o=-0.49)$, and LVEF ( $r h o$ $=-0.32$.

Conclusion: This study suggests that higher body mass index values, "obesity paradox", are not associated with a higher cardiorespiratory level using VO(2)max.

(c) 2014 Sociedad Colombiana de Cardiología y Cirugía Cardiovascular. Published by Elsevier España, S.L.U. This is an open access article under the CC BY-NC-ND license (http://creativecommons.org/licenses/by-nc-nd/4.0/).
\end{abstract}

\section{Introducción}

En 2001, Horwich et al. ${ }^{1}$ acuñaron el término «paradoja de la obesidad» para describir el hallazgo entre el aumento del índice de masa corporal (IMC), con el mejor pronóstico y la evolución clínica de los pacientes con insuficiencia cardiaca. Durante la última década, este hallazgo inesperado se ha relacionado con efectos adversos sobre la estructura cardiaca y la función sistólica y diastólica ventricular, especialmente la izquierda, así como en las enfermedades cardiovasculares, incluyendo la enfermedad coronaria y la hipertensión arterial, que son las causas principales de insuficiencia cardiaca ${ }^{2}$. Para justificar esta paradoja epidemiológica, se han señalado distintas explicaciones, entre otras, que el mayor peso se acompaña de mayor reserva metabólica, lo que permite a estos pacientes enfrentar con mayores posibilidades de supervivencia descompensaciones agudas de una enfermedad crónica. Durante 14 años de seguimiento, en el estudio de Kenchaiah et al. ${ }^{3}$ que incluyó 5.881 participantes del Framingham Heart Study, se demostró que por cada $\mathrm{kg} \cdot \mathrm{m}^{-1}$ de aumento en el IMC, se incrementaba el riesgo de insuficiencia cardiaca en un 5 y $7 \%$ para hombres y mujeres respectivamente, sin importar el valor del IMC al inicio del seguimiento. En este mismo espectro del estado nutricional, Romero-Corral et al. ${ }^{4}$ en un metaanálisis que incluyó 40 estudios y más de 250.000 pacientes con cardiopatía coronaria, se determinó que la mortalidad por insuficiencia cardiaca y enfermedad isquémica cardiaca, no se relacionaba con el incremento del IMC, incluso entre los pacientes con IMC $\geq$ a $35 \mathrm{~kg} \cdot \mathrm{m}^{-1}$.

En cualquier discusión de la «paradoja de la obesidad» en las enfermedades cardiovasculares, existe una relación protectora entre el IMC y la capacidad cardiorrespiratoria aeróbica $(C C R)^{5}$. Se ha descrito que un índice bajo de esta es un potente factor de riesgo cardiovascular, incluso por encima del exceso de peso y grasa corporal $^{5,6}$. Tanto en prevención primaria como en secundaria ${ }^{6}$ la CCR tiene un papel importante en afectar el pronóstico del paciente con insuficiencia cardiaca. Sobre este último, Lavie et al. ${ }^{7}$ demostraron que los pacientes con insuficiencia cardiaca y altos niveles de CCR tienen un pronóstico más favorable en comparación con los sujetos con menor fitness cardiorrespiratorio. Por lo tanto, pacientes con insuficiencia cardiaca con bajo peso (IMC $<20 \mathrm{~kg} \cdot \mathrm{m}^{-1}$ ) y menor CCR (consumo máximo de oxígeno por $\mathrm{VO}_{2 \text { máx }}<14 \mathrm{~mL} \cdot \mathrm{kg} \cdot \mathrm{min}^{-1}$ ) tienen un pronóstico funcional y de calidad de vida particularmente precario. Aunque los niveles más elevados de adiposidad se asocian con mayor riesgo de mortalidad en hombres caucásicos ${ }^{8}$, mayores niveles en la CCR atenúan esta asociación ${ }^{9}$. Recientemente, McAuley et al. ${ }^{10}$, encontraron en 12.417 hombres con sobrepeso y obesidad un incremento en la longevidad entre los sujetos con mayor CCR por $\mathrm{VO}_{2 \text { máx }}$. Este mismo hallazgo fue reportado por Faselis et al. ${ }^{11}$ y Lavie et al. ${ }^{7}$ en pacientes con falla cardiaca, en sujetos sedentarios ${ }^{12}$ y en ciertos tipos de cáncer $^{13}$. De la misma forma, los estudios epidemiológicos «INTERHEART» ${ }^{14}$ e «INTERSTROKE» ${ }^{15}$ han mostrado la contribución protectora de la actividad física al riesgo poblacional atribuible de la enfermedad cardiometabólica en población latinoamericana, posiblemente al efecto endocrino compensador de la masa muscular.

A la fecha, pocos estudios han abordado específicamente esta hipótesis en pacientes con enfermedad cardiovascular documentada ${ }^{5-7,9-11}$. En los trabajos de McAuley et al. ${ }^{7}$ y Faselis et al. ${ }^{11}$ se demostró una asociación inversa entre el IMC y la mortalidad en los pacientes con menor consumo de oxígeno por $\mathrm{VO}_{2 \text { máx }}$. En este contexto, el objetivo de este trabajo fue evaluar la relación entre el estado nutricional por 
Tabla 1 Variables clínicas y antropométricas según la clasificación del índice de masa coporal

\begin{tabular}{|c|c|c|c|}
\hline Variables & $\begin{array}{l}\text { Normopeso } \\
(n=22)\end{array}$ & $\begin{array}{l}\text { Sobrepeso/ } \\
\text { obesidad } \\
(n=18)\end{array}$ & Valor $p$ \\
\hline Edad (años) & $63,7 \pm 15,5$ & $65,2 \pm 12,4$ & 0,34 \\
\hline Peso (kg) & $63,0 \pm 10,6$ & $75,7 \pm 12,6$ & 0,01 \\
\hline Estatura $(\mathrm{cm})$ & $166,5 \pm 10,7$ & $165,1 \pm 12,0$ & 0,60 \\
\hline IMC $\left(\mathrm{kg} \cdot \mathrm{m}^{-1}\right)$ & $23,1 \pm 1,6$ & $28,0 \pm 2,8$ & 0,01 \\
\hline Distancia alcanzada TC6 min (m) & $479,8 \pm 131,2$ & $420,0 \pm 79,7$ & 0,04 \\
\hline Cantidad de pasos (número) & $734,7 \pm 115,4$ & $684,8 \pm 123,1$ & 0,03 \\
\hline $\mathrm{VO}_{2 \text { máx }}\left(\mathrm{mL} \cdot \mathrm{kg}^{-1} \cdot \mathrm{min}^{-1}\right)$ & $11,4 \pm 1,2$ & $10,5 \pm 1,7$ & 0,05 \\
\hline FEVI (\%) & $42,7 \pm 7,8$ & $37,0 \pm 9,0$ & 0,05 \\
\hline
\end{tabular}

Datos presentados en media \pm desviación estándar. Diferencias evaluadas con prueba $U$ de Mann-Whitney.

IMC y los niveles de aptitud cardiorrespiratoria en pacientes con insuficiencia cardiaca.

\section{Materiales y métodos}

\section{Diseño y población}

Durante el segundo semestre de 2013 se realizó un estudio observacional, descriptivo y transversal, en 40 sujetos (31 hombres y 9 mujeres; edad 66,8 $\pm 11,4$ años; IMC $27,4 \pm 4,8$ $\mathrm{kg} \cdot \mathrm{m}^{-1}$; $\mathrm{FEVI} 40,5 \pm 8,3 \%$ ) con diagnóstico médico de insuficiencia cardiaca compensada estadio $C$, clase funcional II-III según la clasificación de la New York Heart Association (NYHA) ${ }^{16}$, con una fracción de eyección del ventrículo izquierdo (FEVI) mayor al 45\%. Se excluyeron aquellos con clase funcional NYHA IV, portadores de arritmias ventriculares complejas, restricción para realizar ejercicio, descompensación aguda de la insuficiencia cardiaca, isquemia miocárdica, claudicación intermitente de miembros inferiores, enfermedades infecciosas o limitaciones neurológicas, ortopédicas o físicas.

\section{Procedimientos}

A todos se les realizó examen clínico, ecocardiograma bidimensional y doppler para estimar la FEVI\% con ecógrafo Acuson Cypress $^{\circledR}$ (Siemens Medical Solutions, Mountain View, California, EE. UU.). Las imágenes fueron analizadas de forma independiente por 2 especialistas en Cardiología con experiencia en esta técnica, quienes desconocían el resultado de las pruebas físicas. La valoración antropométrica comprendió la medición de la estatura y el peso corporal mediante técnicas estandarizadas. La talla se registró en estiramiento con un antropómetro $\mathrm{Krammer}^{\circledR}$ (Holtain Ltd., Crymych Dyfed, RU) de 4 segmentos y $1 \mathrm{~mm}$ de precisión. El peso se midió con balanzas de piso Health-o-Meter ${ }^{\circledR}$ (Continental Scale Corp., Bridgeview, III, EE. UU.) con $100 \mathrm{~g}$ de precisión, calibradas con pesos conocidos. Con estas variables se calculó el IMC en $\mathrm{kg} \cdot \mathrm{m}^{-1}$ y los sujetos se distribuyeron en dos grupos: a) normopeso (IMC entre 18,5 y $24,9 \mathrm{~kg} \cdot \mathrm{m}^{-1}$ ) y b) sobrepeso/obesidad (IMC $\left.\geq 25,0 \mathrm{~kg} \cdot \mathrm{m}^{-1}\right)$.

Para estimar la aptitud cardiorrespiratoria, se realizó el test de caminata de los $6 \mathrm{~min}$ (TC6 min $)^{17}$, tomado en un pasillo de $30 \mathrm{~m}$, incentivando al participante a caminar (no correr), la mayor cantidad de metros durante 6 minutos, en el circuito que se le mostraba previamente. Con la distancia alcanzada en la prueba, se calculó la aptitud cardiorrespiratoria por consumo de oxígeno $\mathrm{VO}_{2 \text { máx }}$ indirecto mediante la ecuación del ACSM: $\mathrm{VO}_{2 \text { máx }}\left(\mathrm{mL} \cdot \mathrm{kg}^{-1} \cdot \mathrm{min}^{-1}\right)=$ metros alcanzados en la prueba TC6 min / $6 \cdot 0,1+3,5$.

La distancia en metros y el número de pasos alcanzados fueron registrados como indicadores de la condición física ${ }^{17}$.

\section{Análisis de datos}

Mediante el test de Kolmogorov-Smirnov se evaluó la distribución de la normalidad para las variables estudiadas. Esto permitió utilizar la prueba no paramétrica $U$ de MannWhitney para muestras independientes. Para estimar la relación entre el IMC, la CCR, la distancia en metros, el número de pasos alcanzados y la FEVI se utilizó el coeficiente de correlación de Spearman (rho). Todas las pruebas se realizaron con el paquete estadístico SPSS 19,0 para Windows (Graphpad Instat, Graphpad Software, University of London, London, UK). Se consideró significativo un valor de $p<0,05$.

\section{Aspectos éticos}

Los Comités de Docencia e Investigación de la Institución participante aprobaron el protocolo que cumple con la Declaración de Helsinki y con la Resolución 008430 de 1993 del Ministerio de Salud de Colombia y se obtuvo consentimiento informado escrito de todos los pacientes.

\section{Resultados}

En la tabla 1 se describen las medias y desviaciones de las características clínicas y antropométricas con base en la clasificación del IMC de la población estudiada $(n=40)$. Se encontraron diferencias significativas por grupos en las variables peso corporal, IMC, distancia alcanzada en el TC6 min, $\mathrm{VO}_{2 \text { máx }}$ y FEVI $(p<0,05)$.

También se encontraron correlaciones negativas en los sujetos con IMC $>25 \mathrm{~kg} \cdot \mathrm{m}^{-1}$ y la distancia alcanzada en el TC6 min $(r h o=-0,50)$, por el número de pasos en el 
Tabla 2 Coeficientes de correlación parcial de Spearman (rho) según el índice de masa corporal y las variables antropométricas, la aptitud cardiorrespiratoria y la fracción de eyección

\begin{tabular}{lcc}
\hline Variables & $\begin{array}{l}\text { Normopeso } \\
(\mathrm{n}=22)\end{array}$ & $\begin{array}{l}\text { Sobrepeso/ } \\
\text { obesidad } \\
(\mathrm{n}=18)\end{array}$ \\
\hline Edad (años) & 0,03 & 0,06 \\
Peso $(\mathrm{kg})$ & $0,45^{*}$ & $0,66^{*}$ \\
Estatura (cm) & $-0,22$ & 0,46 \\
Distancia alcanzada & 0,25 & $-0,50^{*}$ \\
$\quad$ TC6 min (m) & $-0,08$ & $-0,45^{*}$ \\
Número de pasos & $-0,28$ & $-0,49^{*}$ \\
VO ${ }_{2 \text { máx }\left(\mathrm{mL} \cdot \mathrm{kg}^{-1} \cdot \mathrm{min}^{-1}\right)}$ & 0,19 & $-0,32^{*}$ \\
FEVI (\%) &
\end{tabular}

* Correlación bilateral $p=0,05$.

TC6 min $(r h o=-0,45)$, el $\mathrm{VO}_{2 \text { máx }}(r h o=-0,49)$ y en la FEVI $($ rho $=-0,32)($ tabla 2$)$.

\section{Discusión}

Nuestros resultados muestran que los pacientes con sobrepeso/obesidad (IMC > 25,0 $\mathrm{kg} \cdot \mathrm{m}^{-1}$ ) con diagnóstico médico de insuficiencia cardiaca compensada estadio $C$, clase funcional II-III de la NYHA, presentan menores valores de CCR. Este hallazgo coincide con estudios previos que habían puesto de manifiesto este hecho ${ }^{18,19}$. Varias teorías han avanzado en un intento por explicar la «paradoja de la obesidad», incluyendo la hipótesis que ha confirmado que la población obesa es más joven, del efecto protector por el corto período de seguimiento, o por la presencia de factores de confusión como la dieta o el tabaquismo ${ }^{20}$. Si bien estos factores pueden explicar la plausibilidad biológica de la «paradoja de la obesidad», otros estudios epidemiológicos prospectivos han centrado algunas limitaciones en el IMC como una medida que no permite estimar la masa libre de grasa, la cual se relaciona con un mejor estado de salud ${ }^{21,22}$.

Pocos estudios incluyen información acerca de la CCR y la «paradoja de la obesidad» ${ }^{10,13,20}$. En el trabajo que aquí se publica se encontraron correlaciones negativas entre los sujetos con IMC $>25 \mathrm{~kg} \cdot \mathrm{m}^{-1}$ y la distancia alcanzada en el TC6 min (rho $=-0,50$ ), el número de pasos de la prueba de TC6 min (rho $=-0,45)$ y el consumo de oxígeno indirecto por el $\mathrm{VO}_{2 \text { máx }}(\mathrm{rho}=-0,49)$. En este sentido, nuestros datos muestran que menores valores en la aptitud cardiorrespiratoria por $\mathrm{VO}_{2 \text { máx }}$ en la población obesa podrían suponer un factor condicionante al conocido efecto «paradójico protector de la obesidad». Sobre este hallazgo otros autores han señalado que estos individuos podrían presentar precozmente síntomas más graves con menor grado de disfunción sistólica ${ }^{5-10,13,20}$.

La «paradoja de la obesidad» también ha sido descrita en otras enfermedades diferentes a la insuficiencia cardiaca; en pacientes sometidos a una cirugía vascular se encontró mayor mortalidad en el primer mes tras la cirugía en pacientes desnutridos y muy obesos, y menor en sobrepeso y obesidad moderada, incluso ajustado por factores de riesgo como hipertensión o diabetes mellitus ${ }^{23}$. Para explicar los resultados de este estudio, y con base en publicaciones previas, se puede inferir que los pacientes obesos tienen mayor reserva metabólica y, por lo tanto, pueden enfrentar mejor el estrés catabólico inducido por una enfermedad cardiometabólica ${ }^{24}$. Sin embargo, el estado proinflamatorio crónico que caracteriza a los pacientes obesos, el estado de estrés oxidativo, el aumento de citoquinas (TNF- $\alpha$, interleuquinas 1 y 6) y la alteración del equilibrio de las adipoquinas (leptina, adiponectina, etc.), puede explicar el detrimento en la CCR, como lo sugieren Goel et al. ${ }^{9}$.

Sobre este contexto, es muy adecuada la reflexión de Artham et al. ${ }^{22}$ y Zapatero et al. ${ }^{25}$ quienes, al revisar la «paradoja de la obesidad», aconsejan precaución «para no confundir un marcador de riesgo antropométrico con un factor de riesgo» y que, aunque la obesidad es un factor de riesgo de insuficiencia cardiaca, un incremento del peso en estos pacientes mejora la evolución. Por ello, varios autores sugieren otros indicadores antropométricos de adiposidad central, como la medida de la circunferencia de la cintura ${ }^{26}$, porque es fácil y rápida de medir, y porque además, muestra una mejor relación con la grasa intraabdominal medida por tomografía axial computarizada que el IMC ${ }^{27}$. La circunferencia de la cintura muestra una buena asociación con los factores de riesgo cardiovascular, con el riesgo relativo para desarrollar cardiopatía isquémica, hiperinsulinismo, diabetes mellitus tipo 2 y síndrome metabólico, y con la mortalidad cardiovascular de forma independiente al IMC. Sin embargo, estos indicadores presentan algunas limitaciones que podrían conducir, por ejemplo, a clasificar ciertos individuos con masa muscular alta con sobrepeso u obesidad, sin que necesariamente esto ocurra. Investigaciones en países caucásicos muestran de manera inequívoca que la condición física, estimada por la CCR por $\mathrm{VO}_{2 \text { máx }}$ es un importante predictor de mortalidad por causa cardiovascular y por todas las causas en ambos sexos, por lo que no siempre la obesidad debe ser considerada como un factor sano desde el punto de vista metabólico ${ }^{1-4}$. El estudio INTERHEART ${ }^{14}$ también detectó que un bajo nivel de forma física aunado a 9 factores de riesgo, explican en un $90 \%$ el riesgo de experimentar un infarto agudo de miocardio. En el Nurses' Health Study la valoración conjunta del peso corporal y la condición física demostró que el riesgo cardiovascular se incrementa progresivamente en los subgrupos de obesos activos $(R R=2,48)$ frente al grupo de obesos sedentarios $(R R=3,44)^{28}$, mientras que en el Women's Health Study ( $n=38.987$ mujeres durante 11 años de seguimiento), las mujeres obesas y activas presentaron un $R R=1,87$, frente al $R R=2,53$ de las obesas sedentarias ${ }^{29}$.

Finalmente, es preciso tener en cuenta algunos aspectos como limitantes del estudio. Por ejemplo, el IMC fue la única medida antropométrica obtenida, por tanto, no se estimaron otras características de distribución de la grasa corporal, adiposidad o masa libre de grasa. Otras limitaciones como el tamaño de la muestra, las características propias de la población, el diseño del trabajo, el sexo de la muestra y el tipo de muestreo, pueden introducir sesgos. Tampoco se incluyeron otras variables que pueden estar asociadas al riesgo cardiovascular, tales como: etnia, aspectos socio-económicos, nutricionales, sociales y niveles de actividad física directo con acelerómetro. Sin embargo, se observó convergencia de los resultados con datos reportados en otros estudios internacionales $\mathrm{s}^{5-10,13,20}$. 


\section{Conclusiones}

Los resultados muestran que los pacientes con sobrepeso/obesidad (IMC > 25,0 $\mathrm{kg} \cdot \mathrm{m}^{-1}$ ) con diagnóstico médico de insuficiencia cardiaca compensada estadio $C$, clase funcional II-III de la NYHA, presentan menores valores en los indicadores de la CCR estudiados. De acuerdo con estos datos, el análisis conjunto de las mediciones antropométricas y la actividad o forma física pueden ser una alternativa práctica a las limitaciones de la valoración clínica de la obesidad. Como lo proponen Morales y $\mathrm{CoCa}^{30}$, este tipo de mediciones aportarían en la identificación de obesos sedentarios (u obesos con bajo estado físico), subgrupo, en teoría, de mayor riesgo cardiovascular. Es oportuno señalar que se han descrito también paradojas epidemiológicas con otros factores de riesgo cardiometabólicos como hipertensión arterial, dislipidemia y tabaquismo, así como en contextos como insuficiencia renal ${ }^{23}$.

En opinión de los autores, se sugiere incluir la evaluación del parámetro de CCR con pruebas de campo como el TC6 min o test con ergómetros, junto a la determinación convencional de los factores de riesgo tradicionales en el manejo y tratamiento de los pacientes portadores de insuficiencia cardiaca. Se requieren estudios observacionales con mayor tamaño de muestra, y especialmente estudios longitudinales y prospectivos, para constatar los resultados obtenidos en este trabajo.

\section{Conflicto de intereses}

Los autores declaran no tener ningún conflicto de intereses.

\section{Bibliografía}

1. Horwich TB, Fonarow GC, Hamilton MA, MacLellan WR, Woo MA, Tillisch JH. The relationship between obesity and mortality in patients with heart failure. J Am Coll Cardiol. 2001;38:789-95.

2. Lavie CJ, Milani RV, Ventura HO. Obesity and cardiovascular disease: risk factor, paradox, and impact of weight loss. J Am Coll Cardiol. 2009;53:1925-32.

3. Kenchaiah S, Evans JC, Levy D, Wilson PW, Benjamin EJ, Larson MG, et al. Obesity and the risk of heart failure. N Engl J Med. 2002;347:305-13.

4. Romero-Corral A, Montori VM, Somers VK. Association of bodyweight with total mortality and with cardiovascular events in coronary artery disease: a systematic review of cohort studies. Lancet. 2006;368:666-78.

5. Clark AL, Fonarow GC, Horwich TB. Impact of cardiorespiratory fitness on the obesity paradox in patients with systolic heart failure. Am J Cardiol. 2015;15(115):209-13.

6. Swift DL, Lavie CJ, Johannsen NM, Arena R, Earnest CP, O’Keefe $\mathrm{JH}$, et al. Physical activity, cardiorespiratory fitness, and exercise training in primary and secondary coronary prevention. Circ J. 2013;77:281-92.

7. Lavie CJ, Schutter AD, Archer E, McAuley PA, Blair SN. Obesity and prognosis in chronic diseases-impact of cardiorespiratory fitness in the obesity paradox. Curr Sports Med Rep. 2014;13:240-5.

8. Lee CD, Blair SN, Jackson AS. Cardiorespiratory fitness, body composition, and all-cause and cardiovascular disease mortality in men. Am J Clin Nutr. 1999;69:373-80.
9. Goel K, Thomas RJ, Squires RW. Combined effect of cardiorespiratory fitness and adiposity on mortality in patients with coronary artery disease. Am Heart J. 2011:161590-7.

10. McAuley PA, Kokkinos PF, Oliveira RB, Emerson BT, Myers JN. Obesity paradox and cardiorespiratory fitness in 12,417 male veterans aged 40 to 70 years. Mayo Clin Proc. 2010;85: 115-21.

11. Faselis C, Doumas M, Panagiotakos D, Kheirbek R, Korshak L, Manolis A, et al. Body mass index, exercise capacity, and mortality risk in male veterans with hypertension. Am J Hypertens. 2012;25:444-50.

12. McAuley PA, Artero EG, Sui X, Lee DC, Church TS, Lavie CJ, et al. The obesity paradox, cardiorespiratory fitness, and coronary heart disease. Mayo Clin Proc. 2012;87:443-51.

13. Farrell SW, Cortese GM, LaMonte MJ, Blair SN. Cardiorespiratory fitness, different measures of adiposity, and cancer mortality in men. Obesity (Silver Spring). 2007;15:3140-9.

14. Lanas F, Avezum A, Bautista LE. Risk factors for acute myocardial infarction in Latin America: the INTERHEART Latin American study. Circulation. 2007;115:1067-74.

15. O'Donnell MJ, Xavier D, Liu L. Risk factors for ischaemic and intracerebral haemorrhagic stroke in 22 countries (the INTERSTROKE study): A case-control study. Lancet. 2010;376: 112-23.

16. Hurst JW, Morris DC, Alexander RW. The use of the New York Heart Association's classification of cardiovascular disease as part of the patient's complete Problem List. Clin Cardiol. 1999;22:385-90.

17. American College of Sports Medicine (ACSM). Guidelines for exercise testing and prescription. $6{ }^{\text {th }}$. ed. Philadelphia: Lippincott Williams \& Wilkins; 2000.

18. Curtis JP, Selter JG, Wang Y, Rathore SS, Jovin IS, Jadbabaie F, et al. The obesity paradox: body mass index and outcomes in patients with heart failure. Arch Intern Med. 2005;165:55-61.

19. Nigam A, Wright RS, Allison TG, Williams BA, Kopecky SL, Reeder GS, et al. Excess weight at time of presentation of myocardial infarction is associated with lower initialmortality risks but higher long-term risks including recurrent re-infarction and cardiac death. Int J Cardiol. 2006;110:153-9.

20. Lavie CJ, Ventura HO. Analyzing the weight of evidence on the obesity paradox and heart failure-is there a limit to the madness? Congest Heart Fail. 2013;19:158-9.

21. Pengelly CD, Morris J. Body mass index and weight distribution. Scott Med J. 2009;54:17-21.

22. Artham SM, Ventura HO. Insuficiencia cardiaca y la «paradoja de la obesidad»: la historia continúa. Rev Esp Cardiol. 2007;60:1113-7.

23. Niedziela J, Hudzik B, Niedziela N, Gąsior M, Gierlotka M, Wasilewski J, et al. The obesity paradox in acute coronary syndrome: a meta-analysis. Eur J Epidemiol. 2014;29:801-12.

24. Bozorgmanesh M, Arshi B, Sheikholeslami F, Azizi F, Hadaegh F. No obesity paradox-BMI incapable of adequately capturing the relation of obesity with all-cause mortality: An inception diabetes cohort study. Int J Endocrinol. 2014, 2014:282089.

25. Zapatero A, Barba R, González N, Losa JE, Plaza S, Canora J, et al. Influencia de la obesidad y la desnutrición en la insuficiencia cardiaca aguda. Rev Esp Cardiol. 2012;65:421-6.

26. López de la Torre M, Bellido Guerrero D, Vidal Cortada J, Soto González A, García Malpartida K, Hernández-Mijares A. Distribution of waist circumference and waist-to-height ratio by categories of body mass index in patients attended in endocrinology and nutrition units. Endocrinol Nutr. 2010;57: 479-85.

27. Pouliot MC, Despres JP, Lemieux S, Moorjani S, Bouchard C, Tremblay A, et al. Waist circumference and abdominal sagittal diameter: best simple anthropometric indexes of abdominal visceral adipose tissue accumulation and related cardiovascular risk in men and women. Am J Cardiol. 1994;73:460-9. 
28. Li TY, Rana JS, Manson JE, Manson JE, Willet WC, Stampfer MJ, et al. Obesity as compared with physical activity in predicting risk of coronary heart disease in women. Circulation. 2006;113:499-506.

29. Weinstein AR, Sesso HD, Lee IM, Rexrode KM, Cook NR, Manson JE, et al. The joint effects of physical activity and body mass index on coronary heart disease risk in women. Arch Intern Med. 2008;168:884-90.

30. Morales Salinas A, Coca A. Obesity, physical activity and cardiovascular risk: ergo-anthropometric classification, pharmacological variables, biomarkers and «obesity paradox». Med Clin (Barc). 2010;134:492-8. 